\title{
Three-dimensional snow images by X-ray microtomography
}

\author{
Cécile Coléou, ${ }^{1}$ Bernard Lesaffre, ${ }^{1}$ Jean-Bruno Brzoska, ${ }^{1}$ Wolfgang Ludwig, ${ }^{2}$ Elodie Boller ${ }^{2}$ \\ ${ }^{1}$ Centre d'Études de la Neige/Centre National de Recherches Météorologiques/Météo-France, 1441 rue de la Piscine, \\ 38406 Saint-Martin-d'Hères Cedex, France \\ ${ }^{2}$ European Synchrotron Radiation Facility (ESRF), BP 220, 38043 Grenoble Cedex, France
}

\begin{abstract}
For the first time, three-dimensional (3-D) high-resolution images of snow were obtained using X-ray absorption tomography. Images with a spatial resolution of $10 \mu \mathrm{m}$ were taken on four different cylindrical snow samples $(9 \mathrm{~mm}$ high, $9 \mathrm{~mm}$ diameter $)$. About 1000 two-dimensional X-ray absorption images were recorded at angular positions of the object around an axis spanning $180^{\circ}$. An appropriate algorithm was then used for these data to reconstruct a 3-D image. In the case of snow, experimental problems have been solved to prepare the samples and prevent both melting and metamorphism of snow during the experiments. This tomographic method provided 3-D data files from which images of $600^{3}$ voxels were extracted. Several physical parameters of snow microstructure can be processed from these data. Porosity $P$ and discrete local (3-D) curvature $C$ of the grain/pore interface were computed for the four snow samples. Representative elementary volume ( $\mathrm{REV}$, in the sense of porous media) is a relevant index to the significance of the sample size with respect to a given parameter. From each image, the values of $P$ and $C$ are compared for subsamples of different size, as an attempt to assess the REVs for porosity and curvature. Results show that the observed volume of snow is statistically significant to achieve the porosity and the curvature distribution.
\end{abstract}

\section{INTRODUGTION}

Snow is a porous medium, and its internal microstructure (snow grain-size and shape, intergrain bond characteristics, pore structure) acts on its physical properties. This is typically the case for its mechanical properties (Golubev and Frolov, 1998). Most physical processes occurring inside snow are also strictly linked to its microstructure - dry- and wetsnow metamorphism (Colbeck, 1983, 1997) - and also its thermal properties (Arons and Colbeck, 1995). The lack of snow microstructure observations restricts the investigation of snow properties. Therefore snow is considered in many studies as a simple medium like a packing of spherical ice particles. Classical observations such as sieving and photography of grains give quantitative data on grain-size and shape, but do not account for bond properties. Surface sections give more information on the two-dimensional (2-D) structure. The difficulty is then to derive three-dimensional (3-D) properties, especially for parameters which depend on connectivity. More recently, serial cuts have been performed on snow (Good, 1987; Brzoska and others, 1999), but they are time-consuming and the ratio of the sample size to resolution is not really satisfactory (cube of $128^{3}$ voxels of $20 \mu \mathrm{m}$ obtained from 64 section planes and the interpolation of one plane out of two in Brzoska and others, 1999). An alternative way of obtaining information on snow texture is to infer it from other measurements. Information on the pore structure of snow can be deduced from air-permeability and capillaryrise measurements (Jordan and others, 1999) or microstructural and micromechanical parameters using a penetrometer (Johnson and Schneebeli, 1999). These methods provide bulk parameters linked to the microstructure but they do not describe the microstructure itself.
To visualize snow with high resolution, we used X-ray absorption tomography. The method developed to obtain the 3 -D views of snow is described in section 2. In section 3, two important parameters for snow, porosity and local curvature, are calculated on four 3-D images from different snow samples. The results are then discussed in terms of sample size representativity.

\section{METHODOLOGY}

\subsection{X-ray tomography}

The principle of X-ray absorption tomography is to reconstruct the spatial distribution of the linear attenuation coefficient within the object from X-ray projections recorded at different angular settings of the sample. Each element in the recorded projections corresponds to a line integral of the attenuation coefficient along the beam path. Via a standard reconstruction algorithm (filtered back-projection (Herman, 1980)) the 3-D distribution of the attenuation coefficient can be calculated by combining the information for the different angular positions. In our case, 900 projection images spanning $180^{\circ}$ rotation around the vertical axis were recorded for each sample. The reconstructions were performed offline using a standard filtered back-projection software available at the European Synchrotron Radiation Facility (ESRF), Grenoble, France.

\subsection{Experimental set-up}

The experiments were carried out at the ID19 beamline of the ESRF. ID19 is devoted to high-resolution imaging, and features an energy-tunable, parallel X-ray beam of up to $14 \mathrm{~mm} \times 40 \mathrm{~mm}$ cross-section (energy range 7-100 keV; diver- 


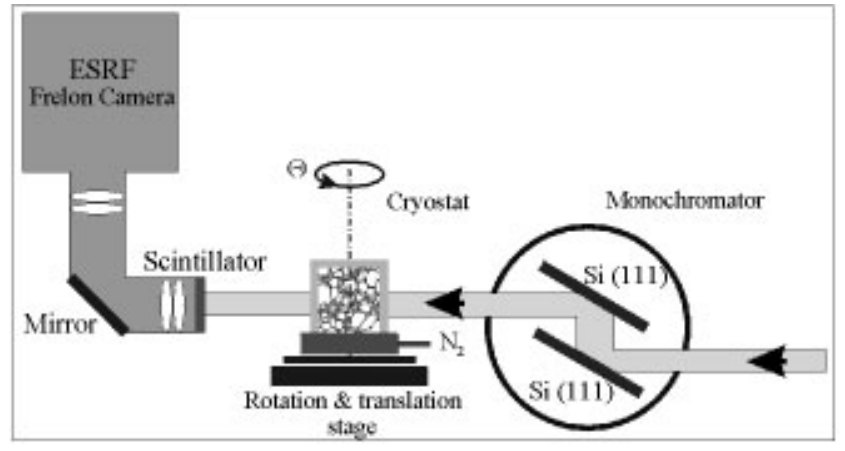

Fig. 1. Diagram of the microtomographic set-up used for snow.

gence $1 \mu \mathrm{rad})$. The cryostat was mounted on a precisionmechanics sample stage (rotation and translation; see Fig. 1), and the projections were recorded with a fast, high-resolution detector system. The latter consists of a powder phosphor screen which is coupled via light optics to a cooled $1024^{2}$ charge coupled device (CCD) camera (Labiche, 1996). For this experiment an effective pixel size of $10 \mu \mathrm{m}$ best met our needs to (a) resolve the shape of individual snow grains and (b) maintain a sufficiently large field of view to observe a statistically significant volume. Typical exposure times for a single radiograph in this configuration are in the order of $1 \mathrm{~s}$, resulting in overall scan times of $15 \mathrm{~min}$.

\subsection{Cryostat}

X-ray microtomography is a well-established technique for visualizing completely diverse objects. The difficulty with our snow samples was to design a special cell able to prevent both melting and metamorphism of the snow sample during the experiment (i.e. for at least $1 \mathrm{~h}$ ). Moreover, the device must not conceal the sample from the beam and must stand up to a $180^{\circ}$ rotation. For this purpose, a refrigerated cell with cylindrical double walls was developed with the collaboration of the Laboratoire de Glaciologie et Géophysique de l'Environnement Grenoble, France (see Fig. 2). Liquid nitrogen

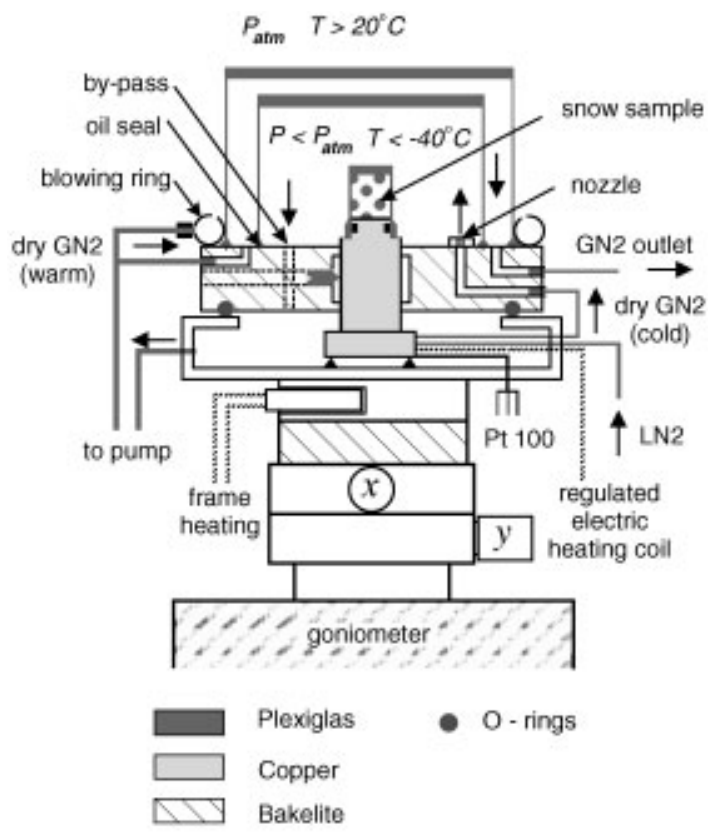

Fig. 2. Details of the cryostat. LN2 indicates liquid nitrogen, and $G \mathcal{N} 2$ gaseous nitrogen.

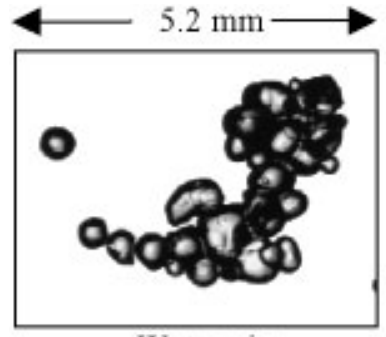

Wet grains

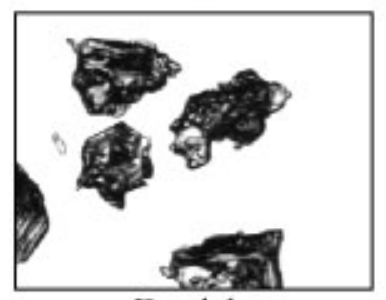

Depth hoar

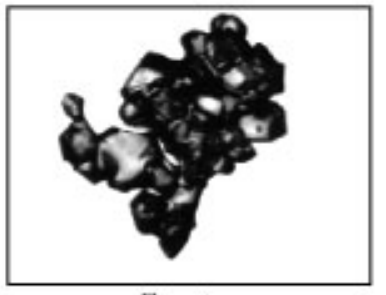

Crust

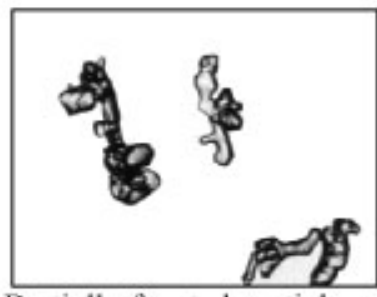

Partially faceted particles
Fig. 3. 2-D views of some grains taken from the four snow samples. For each one, the mean 2-D convex radius of curvature was computed on grain contours from 20 views of this kind.

was evaporated in the bottom of the sample holder, generating a cold and dry gas flow ( $\mathrm{T} \sim-100^{\circ} \mathrm{C}$; dew-point temperature $\mathrm{Td} \ll-40^{\circ} \mathrm{C}$ ), then brought around the Plexiglas sample holder, which ensures a sample temperature lower than $-40^{\circ} \mathrm{C}$. Nitrogen gas was then passed inside the (gas-tight) bottom of the cell for gas warming up to $\left(\mathrm{T} \sim+10^{\circ} \mathrm{C}\right.$; $\mathrm{Td} \ll-40^{\circ} \mathrm{C}$ ) and finally inside and outside the double shell; this prevented both dew outside, and rime inside. To ensure mechanical stability of the sample and to reduce thermal loss, the sample holder was laterally fastened to the cell using a $10 \mathrm{~mm}$ thick Bakelite plate.

\subsection{Sample preparation}

A cubic sample of about $200 \mathrm{~cm}^{3}$ was cut from a snow layer. To prevent sublimation and grain damage during sample machining and handling, the snow texture was strengthened. The technique used was similar to that for thin sectioning (Good, 1987). The sample was soaked by pouring diethyl-orthophthalate (phthalate for short; melting point $-5^{\circ} \mathrm{C}$ ) at $-4^{\circ} \mathrm{C}$. After it was entirely frozen $\left(6 \mathrm{~h}\right.$ at $\left.-20^{\circ} \mathrm{C}\right)$, a lump of about $3 \mathrm{~cm}^{3}$ was extracted and machined, at $-20^{\circ} \mathrm{C}$, into a cylinder $9 \mathrm{~mm}$ in diameter and $9 \mathrm{~mm}$ high. When filled with phthalate, the reconstructed images lacked sufficient contrast to allow automatic processing. Therefore phthalate was removed by rinsing the sample in an isooctane bath at $-2{ }^{\circ} \mathrm{C}$. Isooctane was then drained from the sample by putting the core on blotting paper for $10 \mathrm{~s}$, and finally quickly evaporated under vacuum. To prevent sublimation until the start of the X-ray experiments, the sample was put in a gas-tight cylindrical sample holder and stored in a cool box with $\mathrm{CO}_{2}$ dry ice.

\subsection{Snow characteristics}

Four different snow samples (see Fig. 3) were prepared as described above:

The sample "wet grains" consisted of well-rounded grains, class $6 \mathrm{a}$ according to the International Classification for Snow (Colbeck and others, 1990). They were obtained by soaking a sieved snow sample in water at 


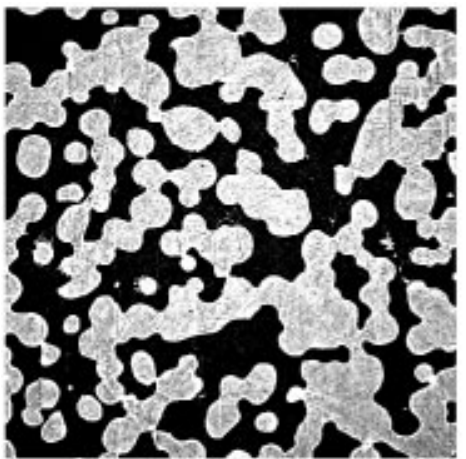

256 grey levels image (4a)

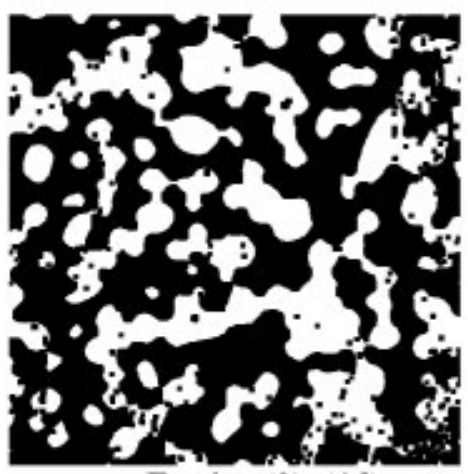

Erosion (3) (4d)

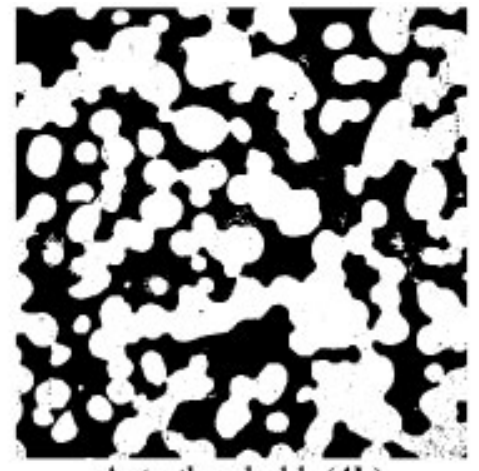

Auto threshold (4b)

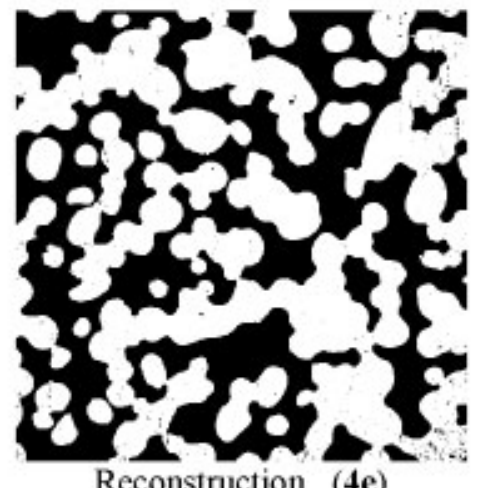

Reconstruction (4e)

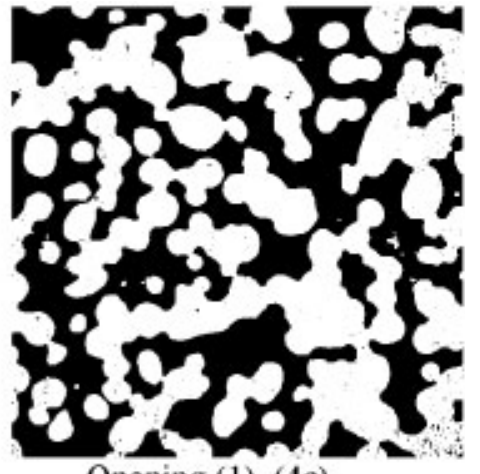

Opening (1) (4c)

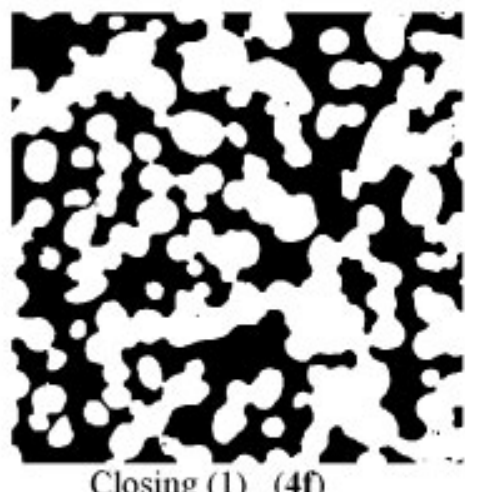

Closing (1) (4f)

Fig. 4. Steps of the processing of the grey-level image of one plane into a binary image. The erosion depth varies between 3 pixels for "wet grains" and 1 for "depth hoar".

$0^{\circ} \mathrm{C}$ and then draining it. Their mean convex radius, computed from 2-D images of grains (Lesaffre and others, 1998), equaled $0.26 \mathrm{~mm}$.

The sample "crust" was collected in the field, at Col de Porte, Chartreuse mountain, France. It consisted of a melt-freeze crust, class 9e, partially faceted. This snow layer was wetted by rain, frozen and then faceted under a large natural temperature gradient. Its mean 2-D convex radius of curvature was $0.25 \mathrm{~mm}$.

The sample "partially faceted particles", class $4 \mathrm{~b}$, resulted from decomposing particles collected in the field and stored at $20^{\circ} \mathrm{C}$ for 1 month, probably under a slight temperature gradient due to storage conditions. It was then sieved and warmed up to $-5^{\circ} \mathrm{C}$ in 2 days. The mean 2-D convex radius was $0.16 \mathrm{~mm}$.

The last sample, "depth hoar", class 5a, was prepared like the previous one, then put into a special device which maintained a large temperature gradient $\left(\sim 100^{\circ} \mathrm{C} \mathrm{m}^{-1}\right)$ for 8 days. Due to experimental problems, this gradient was not applied for the following 3 days. The grains obtained were dense depth hoar; their mean 2-D convex radius was $0.17 \mathrm{~mm}$.

\subsection{Image processing}

Image analysis was performed using classical algorithm on a commercial software (Visilog from NOESIS). Figure 4a presents a view $(600 \times 600$ pixels, 256 grey levels $)$ extracted from one of the 1024 reconstructed planes. Each plane is processed into a binary image where ice is distinguished from pore phase. Since the grey-level images showed sufficient contrast, an automatic threshold using the histogram of the grey-level image was applied (Fig 4b). Some remaining artifacts due to both ill-rinsed phthalate and reconstruction procedure were removed as follows. A first image (Fig. 4c) resulted from an opening procedure, then an erosion of depth 1-3 pixels, depending on sample, was applied (Fig. 4d); the opened image was restored (Fig. 4e) starting from the eroded one as a marker and retrieving only the objects containing a marker. This procedure does not modify the grain's contours but may remove objects whose smallest size is less than 6, 4 or 2 pixels. Finally, small holes inside grains were removed with a closing procedure (Fig. 4f). This may also modify details (maximum size 2 pixels) of the grain's contours. When this process had run on every plane, the binary 3-D data file could be generated. The account for continuity along the $z$ axis was achieved by applying an averaging filter (kernel $3^{3}$, value 1 ) to the data and thresholding the resulting image to 0.5 . Too isolated voxels from grain or pore phase were removed by this procedure. The final result was a smoothed image. The similarity of the binary image overlaid with the grey-level one was visually checked for each plane. The image processes mainly affected small details of the grain's contours. They seemed to be acceptable, although the size of the objects is large compared to the size of the pixels. In our case, "grain-size" was about $0.3-0.5 \mathrm{~mm}$ and pixel size $0.01 \mathrm{~mm}$.

\section{RESULTS}

For the first time, 3-D images of snow at high resolution $(10 \mu \mathrm{m})$ were obtained by X-ray tomography. Image processing was performed on cubes with an edge size of $6 \mathrm{~mm}$, extracted from the cylindrical sample. Figure 5 shows the images obtained from the different snow samples. Each view represents only one-eighth of the volume of the entire processed image. 


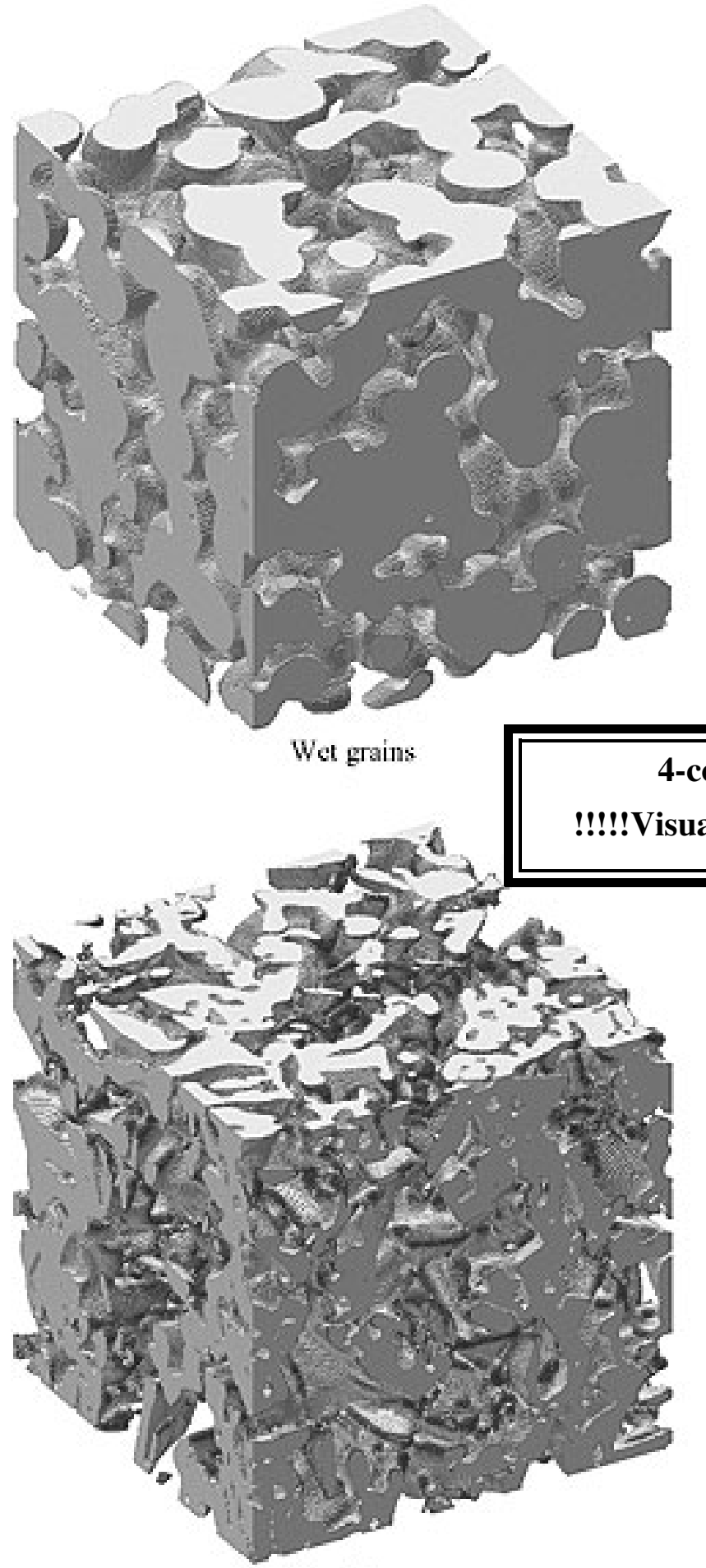

Depth hoar

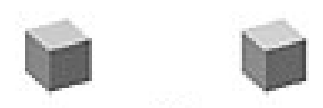

$-25$

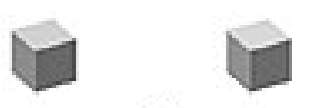

$-15$
$-5$

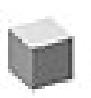

4-colour

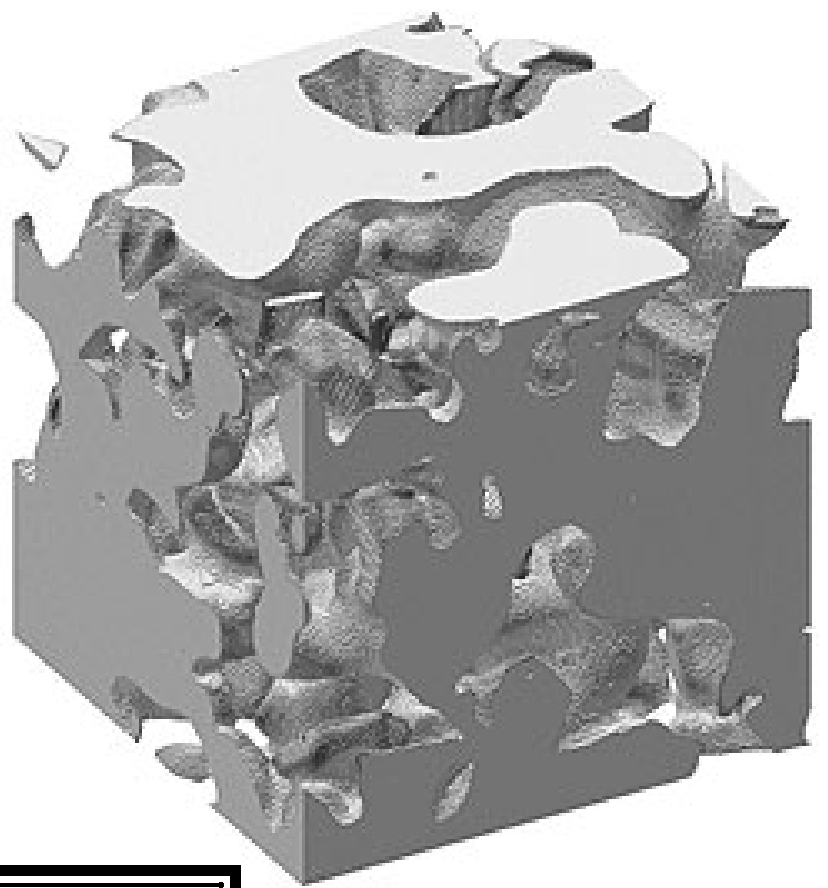

Crust

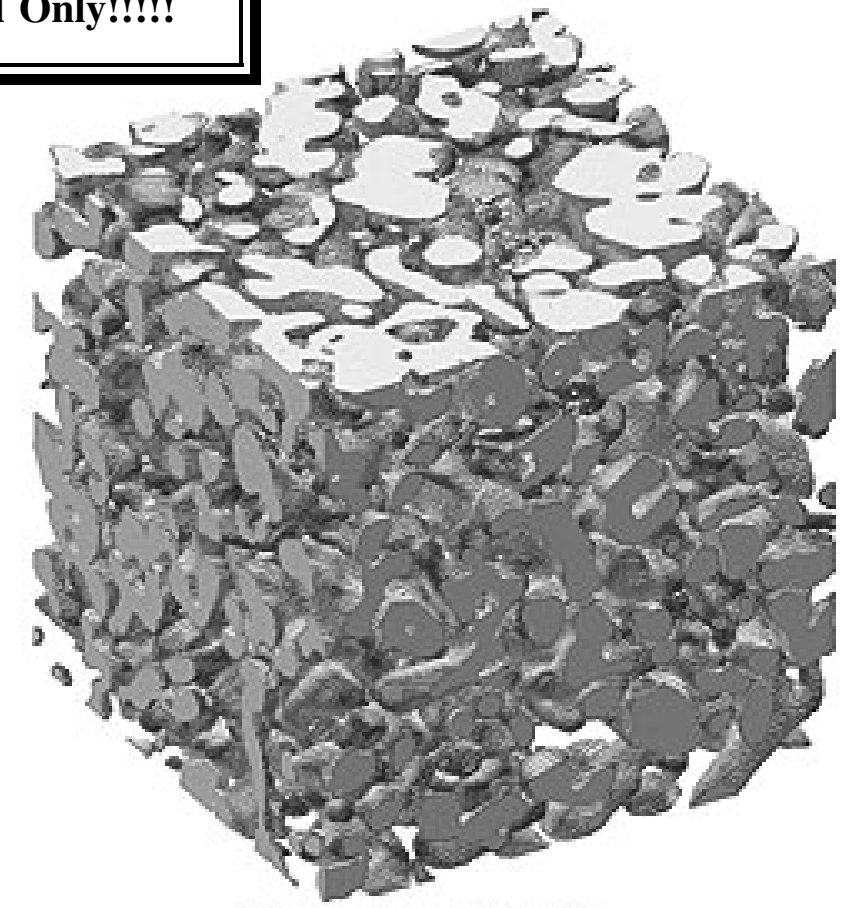

Partially faceted particles

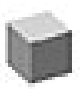

0

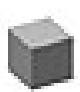

5
15

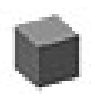

25

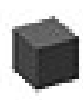

$\mathrm{mm}^{\prime \prime}$

Fig. 5. Subsamples (300 ${ }^{3}$ voxels) of the four 3-D smoothed binary images. According to the computed values of curvature, the convex parts are colored blue, the concave one red; color saturation varies with the absolute value of curvature.

\subsection{Porosity}

Porosity is the simplest descriptive parameter for snow, but the most important for many physical snow properties. Porosity $P$ was determined on the discrete image by counting the voxels in the pore phase. $P=1-\sum_{S} I / N_{\mathrm{v}}$, where $I$ is the value of the voxel in the image ( 1 for ice, 0 for pore), $S$ is a chosen set of voxels, and $N_{\mathrm{v}}$ is the number of voxels in $S$. For each snow sample, porosity was computed on several cubes extracted from the image. In Figure 6, each curve represents the evolution of porosity for one set of embedded cubes where cube size grows from $20^{3}$ to $250^{3}$. The different curves correspond to various separated sets of cubes. When cube size increases, porosity moves towards the mean porosity of the sample at a rate depending on grain type. Whereas the mean porosity of "crust" was achieved with a $10 \%$ precision when the cube's edge is 250 voxels, the same precision was achieved for "partially faceted particles" for much smal- 

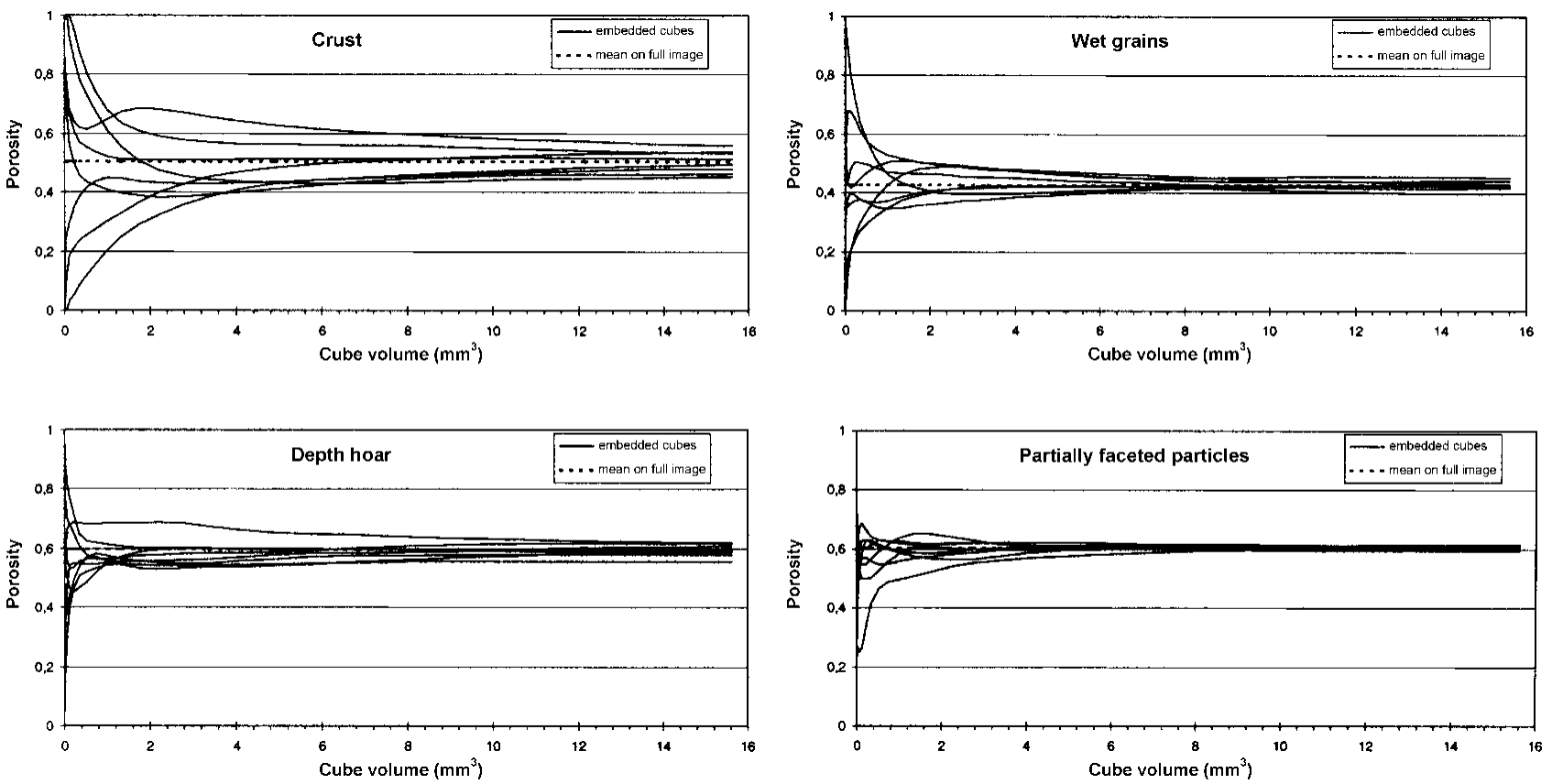

Fig. 6. Evolution of the porosity vs the volume size for each sample. Each curve represents a distinct set of embedded cubes.

ler sampling cubes. Grain-size may explain the differences between the snow samples. It is obvious that small grains require a smaller volume than large grains to show significant porosity.

For the studied samples, picked out from homogeneous snow, the observed volume of $9 \mathrm{~mm}$ high and $9 \mathrm{~mm}$ in diameter is sufficient to reach the porosity. However, this would not be the case in less homogeneous snow (e.g. meltfreeze polycrystals where clusters have a size close to the sample size). Unfortunately, bulk density was not measured, so comparison of the computed porosity with macroscopic density was not possible.

\subsection{Curvature}

Curvature is a central parameter in snow metamorphism. Dry-snow evolution under low temperature gradient and wet-snow evolution are governed by local curvature. In the following the numerical method used for modeling local curvature on discrete images is described. Then the results are presented on the four snow samples, and the determination of the representative elementary volume is discussed. Finally the 3-D convex curvatures are compared with the 2D one from grain's contours.

\section{Model}

The aim was to obtain the local curvature on each point of the surface of the grains. The initial image is the binary image where the values " 1 " represent ice and " 0 " the pores. First of all, it is necessary to define the grain's discrete surface. For every voxel of the object, its background distance is defined as the distance between this point and the closer one belonging to the pore phase. This background distance is computed using the chamfer distance with a kernel $(3,4$, $5)$, i.e. the distance between two voxels in contact with their side, edge and vertex are 3,4 and 5, respectively (Chassery and Montanvert, 1991). The set of voxels whose chamfer distance is 3 defines the grain's surface.

A model has been developed recently for computing cur- vatures on discrete 3-D images (Brzoska and others, 1999). Local curvature on a point $P$ is defined as $C=1 / 2\left[\left(1 / R_{1}\right)+\right.$ $\left(1 / R_{2}\right)$ ], where $R_{1}$ and $R_{2}$ are the radii of curvature computed on two planes orthogonal to each other and containing the normal vector to the surface. The definition of the normal vector provided in Brzoska and others (1999) was based on the association between the voxel from the surface computation and the voxel in the medial axis. It could not be used for $600^{3}$ size images due to processing time. The principle of the new method developed for locating the normal vector is as follows. The discrete chamfer distance map is used to find a voxel $N$ where $(N P)$ is a vector normal to the surface on $P$. Inside a spherical neighborhood of radius $r_{\mathrm{n}}=5$ voxels (with respect to Euclidean distance), $N$ is chosen as the voxel with the maximum background distance. An improvement in the determination of the normal vector would be the optimization of $r_{\mathrm{n}}$ according to the shape of the surface (a larger neighborhood if the surface is smooth, a shorter one if it is rough; this procedure is in development). However, the use of a fixed value of $r_{\mathrm{n}}$ gives a precise enough positioning of the normal vector for the computation of the curvature, and it is convenient. In order to compare the two methods, curvature was computed on a cube of $125^{3}$ voxels, extracted from the sample "partially faceted particles". The mean values of curvature were $5.11 \mathrm{~mm}^{-1}$ for the method using the medial axis and $4.95 \mathrm{~mm}^{-1}$ for the "new" method. The correlation between the two series (121549 values of curvature) was 0.87 . The curvature was computed using a new frame, referenced with the two orthogonal planes. Then the radii of curvature were computed by means of parabolic interpolation of the discrete curves, intersection between grain's contour and the planes of reference.

\section{Application for snow}

Curvature was calculated from 3-D images of $600^{3}$ voxels, on each voxel of the grain's surface, using the method described above. Figure 5 shows the result for the different snow types, the extracted image size being $300^{3}$ voxels. The convex parts are colored blue, the concave one red; color 


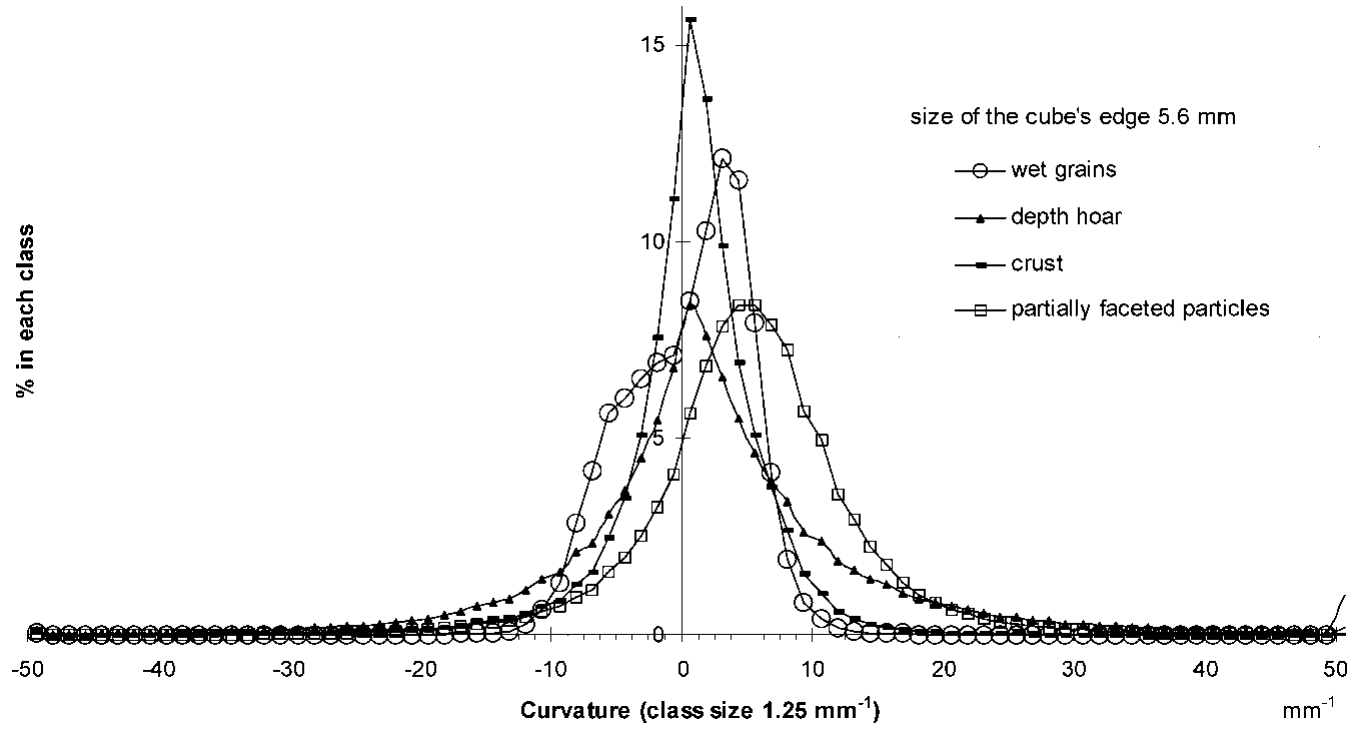

Fig. 7. Histograms of the four curvature distributions on $560^{3}$ voxels cubes.

saturation varies with the absolute value of curvature. The plane zones of "crust" and "depth hoar" have pale colors. For "wet snow", the red parts between the well-rounded grains indicate the place of the refrozen water menisci.

The results in Figure 7 show that the shape of the histogram of curvature is clearly different for each type of grain considered:

"crust" presents a peak in small values, due to the numerous flat zones on the grain's surface;

"wet grains" presents a characteristic bulge in the negative value corresponding to the water menisci (capillary pressure), and a narrow maximum in the positive value;

"partially faceted particles" presents a rather symmetrical histogram centered on a positive value of curvature;

"depth hoar" was derived from the previous sample and then transformed under a large temperature gradient. The resulting histogram is distinctly shifted to the left, corresponding to the growth of the grains and the development of the facets. The numerous negative values are due to the complex shape of the depth-hoar crystals; they should indicate the presence of holes (see Fig. 5) which could appear in the part of the grains that sublimate.
Representative elementary volume

Figure 8 shows several histograms of curvature on the sample "depth hoar". Curvature has been computed on embedded cubes; the size of edge of the cubes varies from 80 to 560 voxels. As soon as the size reaches about 300 voxels, the obtained histograms are similar. This result was verified by computing curvature on separated cubes of size $300^{3}$ voxels. Similar results were obtained for each snow sample. This allows us to conclude that the size of the X-rayed samples is larger than the representative elementary volume for curvature.

\section{Comparison with 2-D results}

A relevant parameter for snow characterization is the histogram of curvature computed on the grain's silhouettes. In Figure 9, the 2-D histograms of convex curvatures of about 50 grains have been superimposed on 3-D histograms. A comparison can be made only for positive curvatures because the bonds between grains, where most negative curvatures are situated, are mostly broken for a 2-D observation. For the samples "crust" and "wet grains", the 3-D histogram is very close to the 2-D one. This is not the case for the other two samples. "Partially faceted particles" is made up of small decomposing particles that tend to lie flat on the observation

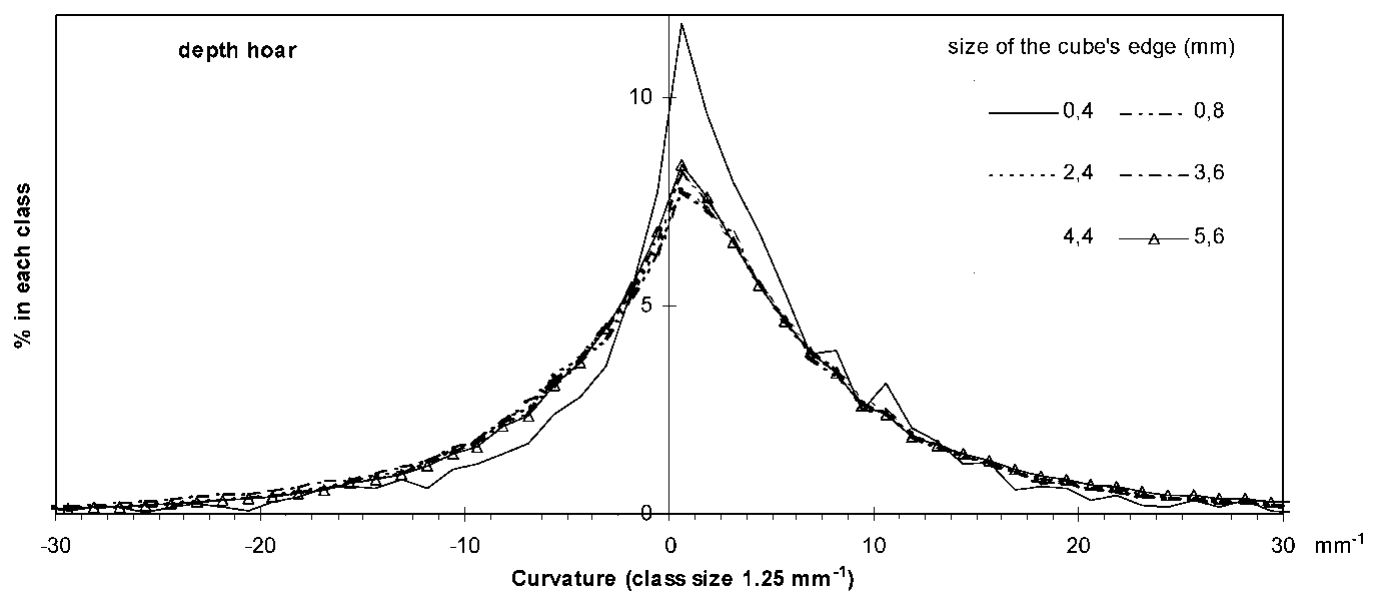

Fig. 8. Variation of the curvature histogram according to the volume of computation. Results for "depth-hoar" sample. 

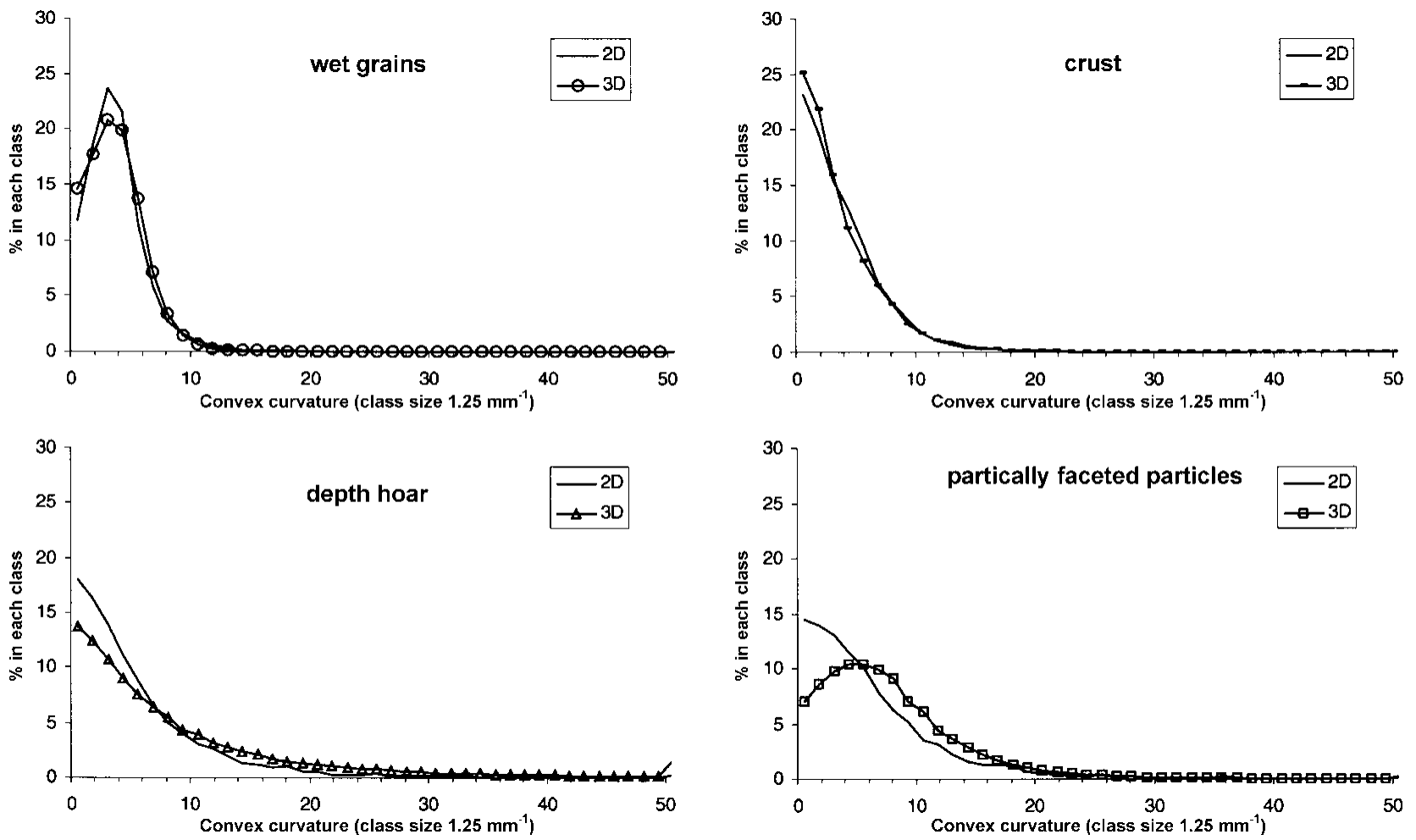

Fig. 9. Comparison between the histogram of 3-D convex curvatures and the 2-D one, for each snow sample.

plate. The edges of grains, the curvature of which is high, are therefore hidden. For "depth hoar" as well, 2-D observation shows only part of the grains. The ridges on the striated grains and the edges of the hollow grains are usually hidden, which decreases the percentage of pixels belonging to highcurvature classes.

\section{DISGUSSION AND GONGLUSION}

The experimental technique described here has proved the feasibility of using X-ray microtomography to visualize snow samples of about $1 \mathrm{~cm}^{3}$ with a resolution of $10 \mu \mathrm{m}$. Presently it can be applied to every snow type cohesive enough to stand up to the rinse of the phthalate before X-ray exposure. Some improvements will soon be tested to visualize more brittle snow types. One way is to replace the filling medium (phthalate) by another chemical which offers a better contrast with ice for the purpose of X-rays. Another way is to replace the imageacquisition method, absorption for the moment, by multiple phase contrast imaging at different distances on snow impregnated with pure phthalate.

Spatial resolution of $10 \mu \mathrm{m}$ is enough to visualize most snow-grain types. Tests on new snow have shown that this is not the case for precipitation particles such as stellars. Future upgrade of the CCD camera to $2048^{2}$ will allow visualization of the same volume with a resolution of $5 \mu \mathrm{m}$.

However, the quality of the 3-D images is very good, and numerous studies can be envisaged that use direct observations of snow microstructure. The calculation of porosity and 3-D local curvature for four different snow samples has shown that the size of the studied sample is statistically representative for these parameters. High-resolution tomographic images will allow further investigation of other features of snow microstructure such as specific area or ice-bond geometry. Moreover, 3-D images could provide valuable data for numerical models in order to investigate complex snow properties like heat conductivity, or complex processes like metamorphism.

\section{ACKNOWLEDGEMENTS}

We thank J. Baruchel and O. Brissaud for fruitful discussions and their help for the experimental set-up, and P. Lamboley who wrote the program for visualizing 3-D data files.

\section{REFERENCES}

Arons, E. M. and S. C. Colbeck. 1995. Geometry of heat and mass transfer in dry snow: a review of theory and experiment. Rev. Geophys., 33(4), 463-493.

Brzoska, J.-B., B. Lesaffre, C. Coléou, K. Xu and R. A. Pieritz. 1999. Computation of 3D curvature on a wet snow sample. Eur. Phys. F. Appl. Phys., 7(1), 45-57.

Chassery, J. M. and A. Montanvert. 1991. Géométrie discrète en analyse d'images. Paris, Edition Hermès.

Colbeck, S. C. 1983. Theory of metamorphism of dry snow. F. Geophys. Res., 88 (C9), 5475-5482.

Colbeck, S. C. 1997. A review of sintering in seasonal snow. CRREL Rep. 97-10.

Colbeck, S. C. and 7 others. 1990. The international classification for seasonal snow on the ground. Wallingford, Oxon, International Association of Scientific Hydrology. International Commission on Snow and Ice.

Golubev, V. N. and A. D. Frolov. 1998. Modelling the change in structure and mechanical properties in dry-snow densification to ice. Ann. Glaciol., 26, 45-50.

Good, W. 1987. Thin sections, serial cuts and 3-D analysis of snow. International Association of Hydrological Sciences Publication 162 (Symposium at Davos 1986 - Avalanche Formation, Movement and Effects), 35-48.

Herman, G. T. 1980. Image reconstruction from projections. New York, Academic Press.

Johnson, J. B. and M. Schneebeli. 1999. Characterizing the microstructural and micromechanical properties of snow. Cold Reg. Sci. Technol., 30(1-3), 91-100.

Jordan, R. E., J. P. Hardy, F. E. Perron, Jr and D. J. Fisk. 1999. Air permeability and capillary rise as measures of the pore structure of snow: an experimental and theoretical study. Hydrol. Processes, 13 (12-13), 1733-1753.

Labiche, J. C., J. Segura-Puchades, D. van Brussel and J. P. Moy. 1996. FRELON camera: Fast REadout LOw Noise. ESRF Newsletter 25, 41-43.

Lesaffre, B., E. Pougatch and E. Martin. 1998. Objective determination of snow-grain characteristics from images. Ann. Glaciol., 26, 112-118. 